\title{
Knockdown of NIR Suppresses Breast Cancer Cell Proliferation via Promoting FOXO3
}

This article was published in the following Dove Press journal: OncoTargets and Therapy

\author{
Bolin Chen (ID) \\ Chengcheng Dong ${ }^{2}$ \\ Fang Wang ${ }^{2}$ \\ Jiacai $\mathrm{Wu}^{2,3}$
}

'Key Laboratory of Cancer Proteomics of Chinese Ministry of Health, Xiangya

Hospital, Central South University, Changsha 410008, People's Republic of China; ${ }^{2}$ School of Biotechnology, Guilin Medical University, Guilin 54II99, People's Republic of China; ${ }^{3}$ School of Pharmacy, Guilin Medical University, Guilin 54I I99, People's Republic of China

Correspondence: Jiacai Wu; Fang Wang Email jiacaiwujc@foxmail.com; fangwang-gl@foxmail.com
Background: Novel inhibitor of histone acetyltransferase repressor (NIR), a corepressor with a novel inhibitor of histone acetyltransferase (INHAT) activity, has been reported to be a negative modulator of p53 and a regulator of the cell cycle in cancer cells. However, the role of NIR in the progression of breast cancer remains elusive.

Materials and Methods: Oncomine database was used to analyze the mRNA levels and prognosis value of NIR in breast cancer. We performed loss-of-function and gain-of-function studies using lentivirus expressing shRNA targeting NIR, enhancer of zeste homolog 2 (EZH2) and forkhead box O3 (FOXO3) or lentivirus expressing NIR or FOXO3, respectively. Cell proliferation and colony formation assays were performed. Coimmunoprecipitation (Co-IP) and immunoprecipitation (IP) were performed to identify the interaction between NIR and polycomb repressive complex 2 (PRC2) subunits. ChIP assay was used to identify the enrichment of NIR, EZH2, H3K27ac and H3K27me3 at the FOXO3 promoter region and the regulation of $\mathrm{H} 3 \mathrm{~K} 27$ modification at the FOXO3 promoter by NIR. Results: High levels of NIR expression were correlated with poor prognosis in breast cancer patients. Knockdown of NIR suppressed the proliferation of breast cancer cells. Mechanically, NIR was recruited by EZH2 to the promoter vicinity of FOXO3 via direct protein-protein interaction. Silencing NIR increased H3K27ac and decreased H3K27me3 levels at the FOXO3 promoter, resulting in enhancing FOXO3 expression. In accordance with this, growth inhibition of breast cancer cells caused by silencing of NIR could be reversed by FOXO3 knockdown.

Conclusion: NIR knockdown inhibited proliferation by switching the H3K27me 3 and $\mathrm{H} 3 \mathrm{~K} 27 \mathrm{ac}$ marks at the FOXO3 promoter to promote FOXO3 transcription, and this effect depends on the physical interaction between NIR and PRC2 in breast cancer cells. Our results suggest that NIR might be a potential target for breast cancer treatment.

Keywords: Breast cancer, NIR, PRC2, EZH2, FOXO3

\section{Introduction}

Breast cancer is one of the most common cancers among females. ${ }^{1}$ Locoregional and systemic treatment, such as surgery, chemotherapy, radiotherapy and molecular therapy, are applied to breast cancer therapy, though the therapies of breast cancer have progressed over the past decades, and early breast cancer is considered potentially curable, it was reported that almost 1.7 million cases were diagnosed and about 522,000 lethal from this disease worldwide in 2012. ${ }^{1,2}$ Numerous tumor suppressor genes and oncogenes were involved in regulating cancer progression, including proliferation. ${ }^{3}$ Therefore, it is an urgent need to investigate the molecular mechanisms and improve the therapeutic strategies of breast cancer. 
Novel INHAT Repressor (NIR) is an epigenetic regulator that represses both basal and activator-stimulated transcription via inhibition of histone acetylation. ${ }^{4}$ In contrast to histone deacetylases, INHATs regulate acetylation mainly through "substrate masking". Moreover, NIR functions as a key regulator of skin development via controlling the expression of essential factors in epidermis development. ${ }^{5}$ Previous studies have shown that NIR functions as a negative regulator of p53-dependent transcription by the direct interaction with $\mathrm{p} 53 .{ }^{4}$ NIR has also been shown to repress the expression of $\mathrm{p} 63$ by restricting H3K18ac at the p63 promoter. $^{6}$ NIR has been found to play important functions in lymphocytes development by cooperating with $\mathrm{p} 53$ to impose checkpoint for the generation of mature $\mathrm{B}$ and $\mathrm{T}$ lymphocytes. ${ }^{7}$ But the role of NIR in breast cancer is unclear.

Accumulating evidence suggests that histone modifications and their associated chromatin-modifying enzymes play causal roles in cancer development. ${ }^{8}$ Polycomb repressive complex 2 (PRC2), one of the best characterized Polycomb group (PcG) protein complexes, plays important roles in gene repression through catalyzing histone H3K27 methylation, and dysregulation of PRC2 has been observed in different cancer types. ${ }^{9}$ PRC2 contains the three core subunits, Enhancer of zeste homolog 2 (EZH2), Embryonic ectoderm development (EED), and Suppressor of zeste (SUZ12), which are essential for PRC2 activity. ${ }^{10}$ Depletion of any core subunit in mice resulted in embryonic lethality due to disturbance of the expression of PcG target genes via globally changing H3K27 modification in the embryonic stem cells. ${ }^{11} \mathrm{EZH} 2$, the catalytic subunit of the PRC2, is involved in the epigenetic silencing of tumor suppressor genes in cancer, and aberrant expression or mutations of EZH2 is found in various cancer types. For example, the elevated expression of EZH2 is associated with tumor progression and poor prognosis in breast cancer. ${ }^{12,13} \mathrm{On}$ the contrary, loss-of-function mutations in EZH2 are associated with poor prognosis in myeloid neoplasms. ${ }^{14}$ Therefore, the dysregulation of EZH2 function and expression is complex and cancer type-dependent. The activity of EZH2 is mainly modulated by post-translational modifications and the interaction proteins of EZH2. For example, phosphorylation at T345 and T487 of EZH2 by CDK1 and CDK2 was shown to regulate the epigenetic gene silencing in breast and prostate cancer, ${ }^{15,16}$ and EZH2-K348 acetylation greatly enhanced the repression of EZH2 target genes. ${ }^{17}$ Foxhead box O3 (FOXO3), a known tumor suppressor, is a member of the Forkhead box family. ${ }^{18}$ It has been reported that FOXO3 is crucial in the progression of cancer cells, including cell proliferation, aggressive and apoptosis, low FOXO3 expression is correlated with high tumor stages and poor survival in breast cancer patients, and $\mathrm{FOXO} 3$ is negatively regulated by EZH2 in both hepatocellular carcinoma and breast cancer. ${ }^{18-21}$

In this study, we investigated the functions of NIR in the regulation of breast cancer. We found that high levels of NIR were associated with poor prognosis in breast cancer patients, silencing NIR inhibited proliferation and colony formation in breast cancer cells, and this effect is at least in part, mediated by upregulating the expression of FOXO3. Furthermore, we discovered that NIR directly interacted with EZH2 in the PRC2 complex to regulate H3K27 modification at the promoter of FOXO3, representing a novel mode of action by which NIR controls the levels of tumor suppressor gene expression.

\section{Materials and Methods}

\section{Cell Culture and Cell Line Construction}

The human breast cancer cell lines MDA-MB231, HCC1937 and MCF7, and embryonic kidney cell line, HEK-293T, were purchased from the Type Culture Collection of the Chinese Academy of Science (Shanghai, China). All cell lines were maintained in Dulbecco's modified eagle's medium and supplemented with $10 \%$ fetal bovine serum (Gibco, USA) at $37{ }^{\circ} \mathrm{C}$ and $5 \% \mathrm{CO}_{2}$. For doxycycline-inducible expression, the medium was supplemented with $1 \mu \mathrm{g} / \mathrm{mL}$ doxycycline (SigmaAldrich) or PBS as the vehicle control.

Lentivirus plasmids pWPT and pLVTHM were presented by Prof Trono Didier. The lentivirus was packaged and used to infect cells according to previous methods. ${ }^{22}$ The oligo DNA for silencing NIR (GTGGTACAGGCGTTCCGAG) or EZH2 (GAAAGAACGGAAATCTTAAA) was cloned into pLVTHM for lentivirus packaging and the indicated cell lines were constructed by lentivirus infection. Lentivirus, for silencing of FOXO3, was purchased from Jikai Gene Chemical Technology Co. LTD (Shanghai, China). For the rescue of NIR expression, NIR genes were first mutated at shNIR targeted position, to replace three amino acid codes, and then this engineered NIR gene was cloned into a pWPT lentivector using a PCR cloning method. Ectopic expression of FLAGtagged FOXO3 was achieved by using pWPT lentivirus vector.

\section{Antibodies}

Antibodies used were as follows: anti-NIR (HPA044258, Atlas Antibodies), anti-EZH2 (\#5246, Cell Signaling Technology, CST), anti-SUZ12 (\#3737, CST), antiFOXO3 (\#2497, CST), anti-EZH1 (\#62,083, CST), anti- 
EED (03-196, Millipore), anti- $\beta$-actin (\#3700, CST), antiFlag (2054-1-AP, Proteintech), anti-Flag M2 (F3165, Sigma), anti-HA (66,006-2-Ig, Proteintech), anti-Histone H3K27me3 (ab6002, Abcam), anti-Histone H3K27ac (ab4729, Abcam), Rabbit mAb IgG (\#3900, CST), Mouse mAb IgG (\#5415, CST).

\section{Real-Time Quantitative PCR Assay}

The total RNA of the cell lines was extracted by using Eastep $^{\circledR}$ Super Total RNA Extraction Kit (\#LS1040, Promega Corporation), and $1 \mu \mathrm{g}$ of total RNA was reversetranscribed to cDNA with Revert Aid First Strand cDNA Synthesis Kit (\#K1622, Thermo Fisher Scientific). The quantitative PCR was performed by using the FastStart Universal SYBR Green Master (Rox) (Roche) in 8-well strips on the 7500 Fast Dx Real-Time PCR System (ABI). The mRNA level of L19 was treated as an internal control, and all samples were assayed in triplicate for three independent experiments. The primer sequences (from $5^{\prime}$ to $3^{\prime}$ ) were listed as follows, EZH2 forward: AATCA GAGTACATGCGACTGAGA, reverse: GCTGTATCCTT CGCTGTT TCC; NIR forward: AAGCAACGCCTCACT CCAAA, reverse: ACTGTCCGTGACCTGGAATTT; FOXO3 forward: TCACGCACCAATTCTAACGC, reverse: CACGGCTTGCTTACTGAAGG; L19 forward: AAAACAAGCGGATTCTCATGGA, reverse: CCTC TTGGCCGTTTTTCTCCA.

\section{Cell Proliferation Assay and Colony Formation Assay}

The cells were induced with doxycycline (Dox) or vehicle for $4 \mathrm{~d}$, for the subsequent assays. For cell proliferation assay, $2.0 \times 10^{5}$ cells were plated per $60 \mathrm{~mm}$ plate, the cells were collected and counted daily to analyze the cell proliferation. For colony formation assay, 2000 cells were seeded in a $60 \mathrm{~mm}$ plate and cultured for $12-15 \mathrm{~d}$, until the cell colonies became visible. The colonies were fixed with $25 \%$ acetic acid in $75 \%$ anhydrous methanol at room temperature for $20 \mathrm{~min}$, and then stained with Giemsa stain (Sigma) for colony counting.

\section{Co-Immunoprecipitation (Co-IP)}

Cells were lysed in immunoprecipitation buffer $(50 \mathrm{mM}$ Tris- $\mathrm{HCl} \mathrm{pH}$ 7.5, $1 \mathrm{mM}$ EGTA, $1 \mathrm{mM}$ EDTA, $150 \mathrm{mM}$ $\mathrm{NaCl}, 0.5 \%$ Triton $\mathrm{X}-100)$ containing protease inhibitor (Cocktail) on ice for $30 \mathrm{~min}$, then sonicated ( 5 cycles, 10s $\mathrm{ON} / 30 \mathrm{~s}$ OFF) in a Bioruptor (Diagenode). The cell lysates were centrifuged at 15,000 rpm for $10 \mathrm{~min}$. Per Co-IP, 300 $\mu \mathrm{L}$ of protein extracts were incubated with $0.5 \mu \mathrm{g}$ specific antibodies or IgG for $2 \mathrm{hrs}$ on ice, then $30 \mu \mathrm{L}$ protein $\mathrm{G}$ beads slurry (Pierce) was added to the mixtures, and incubated for $1 \mathrm{hr}$ at $4^{\circ} \mathrm{C}$. After washing five times with the cell lysis buffer, the immunoprecipitated complexes were eluted and detected by Western blot.

\section{Immunoprecipitation (IP)}

The proteins were transcribed and translated in vitro by using TNT $^{\circledR}$ SP6 Coupled Wheat Germ Extract System (\#L4130, Promega). The proteins were pretreated with Benzonase and RNase to degrade DNA and RNA. For each immunoprecipitation assay, $20 \mu \mathrm{L}$ of each relevant translated product was diluted in $200 \mu \mathrm{L}$ of IP buffer containing $0.2 \% \mathrm{BSA}$ and the indicated two proteins were mixed and incubated on ice for $2 \mathrm{hrs}$. After binding, $0.5 \mu \mathrm{g}$ of indicated antibody and $30 \mu \mathrm{L}$ protein $\mathrm{G}$ beads slurry was used to IP interaction proteins. After critical washing the beads and elution, the immunoprecipitants were detected by Western blot.

\section{Chromatin Immunoprecipitation (ChIP)}

The protocol of ChIP is according to previous reports. ${ }^{23}$ MDA-MB231 cells were cross-linked with $1 \%$ formaldehyde at room temperature for $10 \mathrm{~min}$ and sheared by sonication. Anti-HANIR, -EZH2, -H3K27me3, -H3K27ac antibodies, and $\mathrm{IgG}$ were used for immunoprecipitation, and the $1 \%$ input served as control. The purified DNA samples were analyzed by qPCR with the specific primers. The ChIP-qPCR primer sequences (from 5' to $3^{\prime}$ ) of FOXO3 are according to the previous report, ${ }^{20}$ and the sequences are as follows: P1 forward: GAATGCTGGCATTTCCTCTC, reverse: CACAAATTGCCTCGACCTTT; P2 forward: ACTTGAAGCCGAGTTGTGG, reverse: GGGGCTCT GACTGCTTACTG; P3 forward: GCGTGCGTTTG TTTATGTT, reverse: TTTGTACGCGTCGAACTAGC.

\section{Public Data Acquisition}

The Curits breast dataset from Oncomine database (https:// www.Oncomine.org) was used to explore the mRNA levels of NIR in breast cancer.

\section{Statistical Analysis}

Statistical analysis was performed with GraphPad Prism Version 8.0 (GraphPad Software Inc., La Jolla, CA, USA). Survival analysis was estimated by the Kaplan-Meier with the Log-rank test. Gray image analysis of Western blot 
was performed by using Image J (NIH, USA). The statistical difference was performed by two-tailed Student's $t$-test, one-way analysis of variance (ANOVA) or twoway ANOVA test. Results were presented as mean $\pm \mathrm{SD}$ of three or more independent experiments, $p$-values $\leq 0.05$ were considered statistically significant.

\section{Results}

\section{High mRNA Levels of NIR in Breast Cancer are Associated with Poor}

\section{Prognosis}

NIR, which was first known as a novel inhibitor of histone acetyltransferase (INHAT), can regulate the cell cycle of kinds of cancer cell lines, ${ }^{24}$ but the function of NIR in breast cancer is still unknown. To investigate the clinical relevance of NIR in breast cancer progression, we first analyzed the mRNA levels of NIR by using Curits breast dataset which includes 2 , 136 clinical cases from the Oncomine database (www.onco mine.org), ${ }^{25}$ we found that the average levels of NIR were higher in invasive ductal breast carcinoma as compared to the normal breast tissues (Figure 1A). Furthermore, KaplanMeier survival analysis showed that higher mRNA levels of NIR were linked to poorer overall survival than those with lower NIR mRNA levels in invasive ductal breast carcinoma patients (Figure 1B). These results indicate that high levels of NIR might contribute to poor prognosis in breast cancer.

\section{Knockdown of NIR Represses Cell Proliferation and Colony Formation of Breast Cancer Cells}

Our previous study showed that upregulation of NIR was associated with poor survival in breast cancer patients, but how NIR functions in breast cancer cells are still unknown. To investigate the role of NIR in the regulation of breast cancer cell growth, a doxycycline (Dox) inducible NIR knockdown breast cancer cell line, MCF7-Tet-onshNIR, was constructed. Cells treated by Dox for 0, 1, 2, 3, 4, and 5 days were harvested for Western blot using a NIR antibody to evaluate the silencing efficacy. The results showed that more than $70 \%$ of NIR protein was knocked down in MCF7-Tet-on-shNIR cells after 4 days of Dox treatment (Supplementary Figure S1A). The growth curves of MCF7-Tet-on-shNIR cells with or without Dox treatment were measured. The same number of cells was seeded after 4 days treatment with Dox or vehicle, and the cell numbers were counted on the indicated days, respectively. The cell growth curves showed that the cell proliferation was significantly inhibited by NIR silencing on the third and fourth day (Figure 2A and B). MCF7-Tet-on-shNIR cells were parallelly cultivated for colony formation assay with or without Dox treatment. As shown in Figure $2 \mathrm{C}$ and $\mathrm{D}$, the colony numbers of MCF7-Tet-on-shNIR cells treated with Dox
A

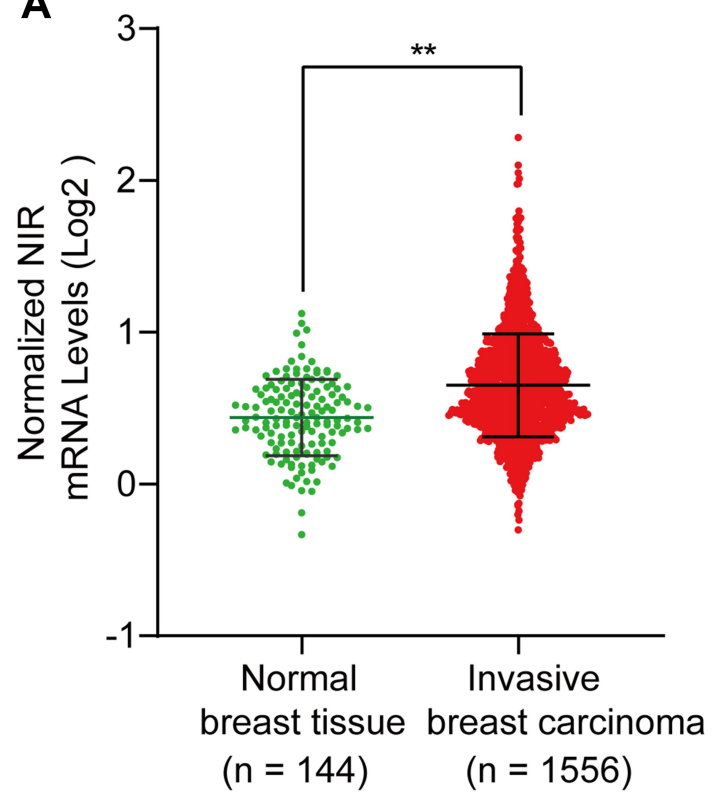

B

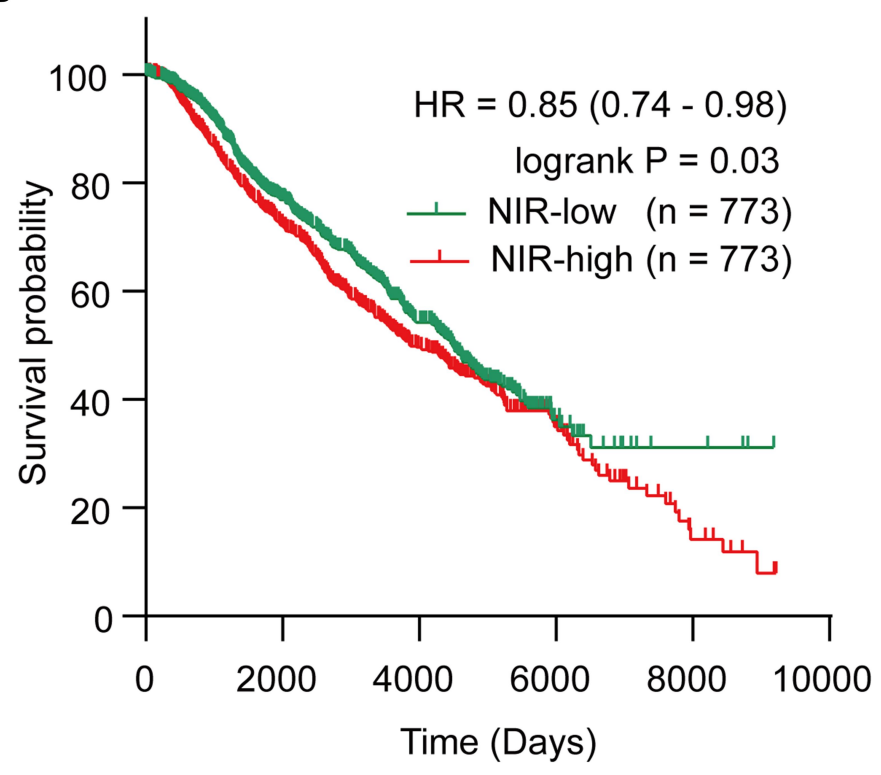

Figure I High level of NIR expression is associated with poor survival in breast cancer patients.

Notes: The Curits breast dataset from Oncomine was used. (A) Analysis of the mRNA levels of NIR between normal breast tissues and ductal invasive breast carcinoma. (B) Survival analysis of NIR mRNA levels in ductal invasive breast carcinoma (NIR-low, $n=773$; NIR-high, $n=773)$. ** denotes $p<0.01$. 
A
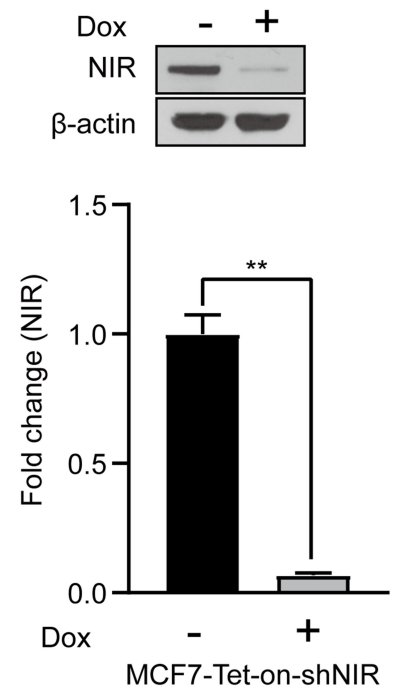

B

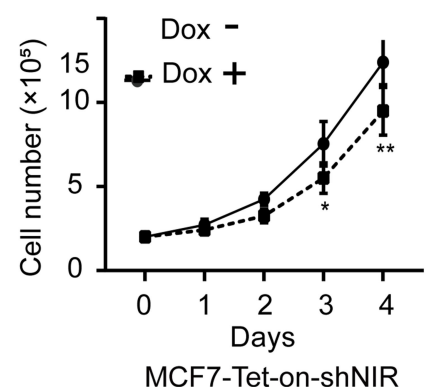

C

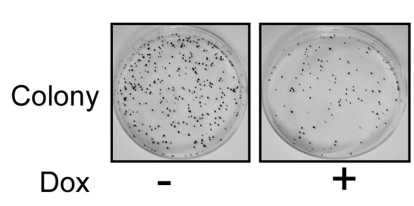

MCF7-Tet-on-shNIR

D

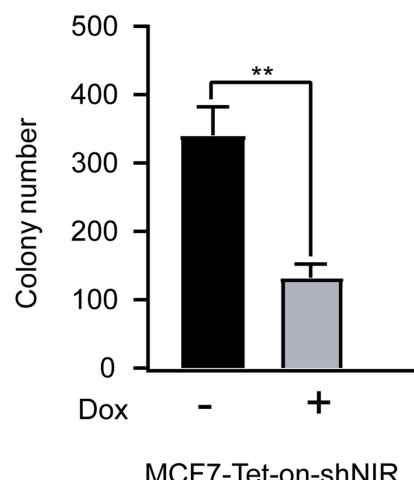

E
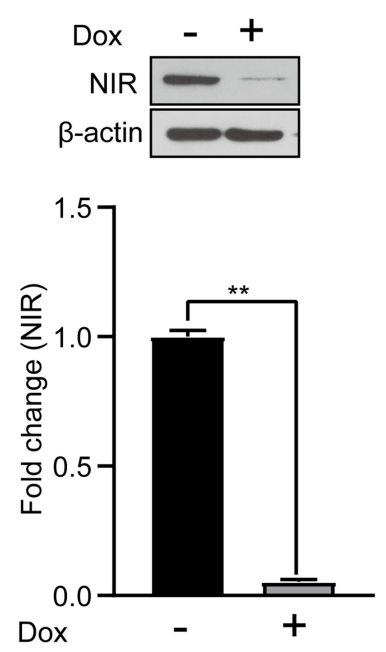

MDA-MB231-Tet-on-shNIR

F

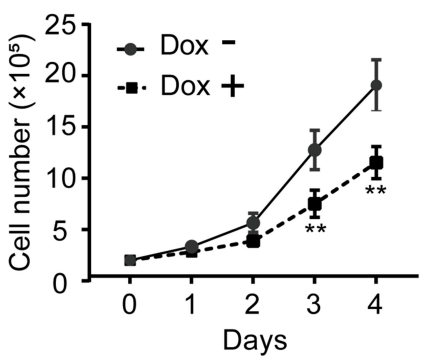

MDA-MB231-Tet-on-shNIR

G

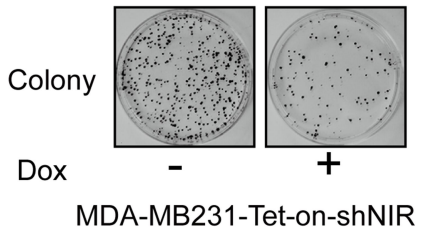

H

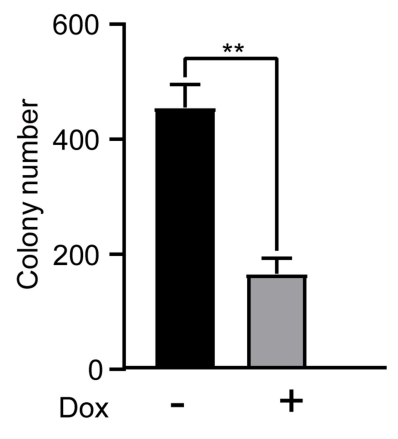

MDA-MB231-Tet-on-shNIR
I
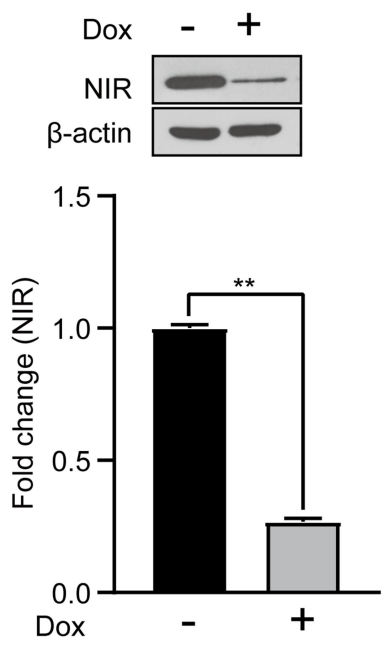

HCC1937-Tet-on-shNIR

J

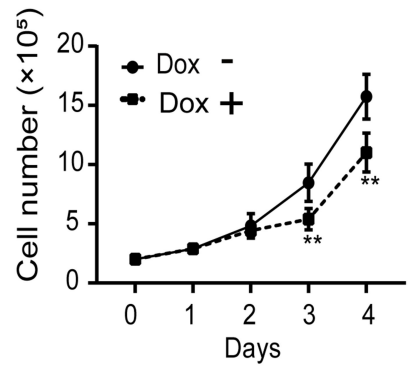

HCC1937-Tet-on-shNIR

K

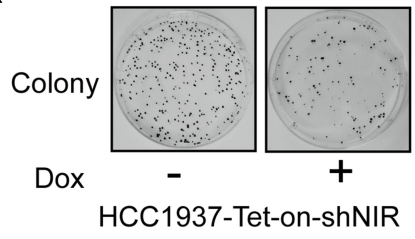

$\mathbf{L}$

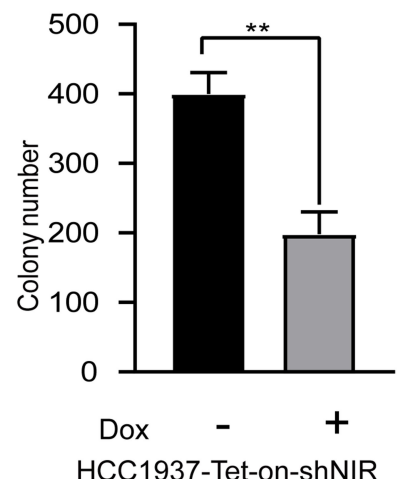

Figure 2 Knockdown of NIR (novel INHAT repressor) represses cell proliferation and colony formation in breast cancer cells.

Notes: (A) Western blot showed that NIR was strongly silenced in MCF7-Tet-on-shNIR cells after Dox treatment for 5 days. (B) MCF7-Tet-on-shNIR cells were treated with or without Dox for 4 days and the same number of two types of cells were seeded to test the growth curves. The proliferation of MCF7 cells was significantly inhibited by silencing NIR. (C) Giemsa staining showed colony formation in Dox-induced and non-induced MCF7-Tet-on-shNIR cells. (D) The colony formation of MCF7 cells was significantly suppressed after silencing NIR. (E) NIR was silenced in MDA-MB23 I-Tet-on cells. The cell proliferation (F) and colony formation $(\mathbf{G}$ and $\mathbf{H})$ were detected in Dox treated or untreated cells, separately. (I-L) Silencing of NIR suppresses HCCI 937 cell proliferation and colony formation abilities. The Western blot images were analyzed by Image J (NIH, USA). The results are presented as averages of three independent experiments. Error bars represent standard deviation and two-tailed unpaired Student's $t$-test was performed. $*$ denotes $\mathrm{p}<0.05$, $* *$ denotes $\mathrm{p}<0.0 \mathrm{I}$. 
were significantly lower than those without Dox treatment. Since NIR has been shown to regulate p53 function, and MCF7 cells express wild-type p53, ${ }^{4}$ we re-examined NIRmediated growth inhibition in a p53-mutant breast cancer cell line, MDA-MB231, to exclude the possibility of the involvement of p53. Similar to the findings in MCF7-Teton-shNIR cells, more than $70 \%$ of NIR protein was reduced after 4-day Dox treatment (Supplementary Figure S1B) in the MDA-MB231-tet-on-shNIR cells. As shown in Figure 2E-H, NIR silencing could also significantly inhibit MDA-MB231 cell proliferation and colony formation. These results were further confirmed in another p53-mutated breast cancer cell line, HCC1937 (Supplementary Figure S1C, Figure 2I-L). Collectively, our results suggest that NIR is essential for cell proliferation in a p53-independent manner.

To exclude the off-target effect of shNIR, the NIR expression was rescued by ectopic expression of Flagtagged NIR which could escape the shRNA-mediated silencing of NIR, in the MDA-MB231-Tet-on-shNIR cells. Western blot results showed that the endogenous NIR was efficiently knocked down by shRNA, and the total NIR expression level was restored by infecting the cells with pWPT-Flag-NIR virus (Figure 3A). Restoration of NIR was sufficient to rescue the growth in NIR knockdown MDA-MB231 cells (Figure 3B-D). These results further demonstrate that NIR is an essential regulator of breast cancer cell proliferation.

\section{NIR Associates with the PRC2 Complex}

Although the previous studies had shown functional regulation of $\mathrm{p} 53$ by NIR, ${ }^{4}$ the regulation of cell proliferation by NIR in p53-mutant expressing breast cancer cell proliferation triggered us to explore additional mechanisms underlying NIR function. Dysregulation of PRC2 complex has been implicated in breast cancer development. ${ }^{10} \mathrm{EZH} 2$ is the core enzymatic subunit of PRC2, which maintains the gene in a transcriptionally silent state by trimethylating histone $\mathrm{H} 3$ on lysine $27 .{ }^{26}$ Since PRC2 has been reported to physically associate with $\mathrm{HDAC} 1$ and HDAC2, ${ }^{9}$ NIR inhibits histone acetylation in HDACindependent mechanism, we postulated if NIR functionally cooperate with $\mathrm{PRC} 2$ to regulate histone acetylation. To test this hypothesis, we stably re-expressed Flag-tagged NIR in MDA-MB231-shNIR cells and immunoprecipitated NIR interacting proteins from whole-cell lysates using anti-Flag antibody. Western blot results showed that the PRC2 core subunits (EZH2, EED, and SUZ12) were co-precipitated with NIR (Figure 4A), whereas EZH1 was not detected (Figure 4A). These results indicated that NIR specifically interacted with the EZH2, but not the EZH1-containing PRC2 complex. Furthermore, the interaction between NIR and PRC2 complex was confirmed by co-immunoprecipitation using anti-Flag (from MDAMB231-HANIR-FlagEED cell lysates) anti-EZH2, or SUZ12 (from MDA-MB231-HANIR cell lysates) antibody (Figure 4B-D). These results strongly support the specific association between NIR and PRC2 complex.

To identify the subunit of the PRC2 complex responsible for interaction with NIR, individual FLAG-tagged EZH2, EED, and SUZ12, and HA-tagged NIR proteins were expressed by in vitro transcription and translation. Individual FLAG-tagged PRC2 subunit was normalized and immobilized on beads using anti-Flag M2 antibody and then incubated with the in vitro translated HA-NIR protein, respectively. Upon normalization of the equal amount of input protein, the immunoprecipitation results showed that neither SUZ12 nor EED bound with NIR; however, EZH2 interacted with NIR (Figure 4E). These results indicate that NIR directly associates with PRC2 complex via EZH2.

\section{Silencing EZH2 or NIR Promotes FOXO3 Expression in Breast Cancer Cells}

A previous study had shown that $\mathrm{EZH} 2$ is a negative regulator of $\mathrm{FOXO} 3$, and inhibition or depletion of $\mathrm{EZH} 2$ led to induction of $\mathrm{FOXO} 3$ expression through reducing the $\mathrm{H} 3 \mathrm{~K} 27$ trimethylation level at the FOXO3 promoter in breast cancer cells. ${ }^{20}$ Moreover, decreasing the level of EZH2 inhibits the cell proliferation and clonogenicity abilities of MCF-7 and MDA-MB231 cells. ${ }^{27}$ Given the direct interaction between NIR and EZH2, we tested if NIR could regulate FOXO3 expression by cooperating with EZH2 in MDA-MB231 cells. FOXO3 expression levels were detected by RT-qPCR and Western blot in EZH2-silenced or NIR-silenced MDA-MB231-Tet-on cells. Our results showed that knocking down either EZH2 or NIR promoted the expression of FOXO3 at both mRNA and protein levels (Figure 5A-D). EZH2 and NIR does not seem to reciprocally regulate each other as detected by the mRNA and protein expression levels (Figure 5). The same results were obtained in HCC1937 cells (Figure 5E and Figure 4H). These results suggested that FOXO3 was regulated by NIR and EZH2 in a coordinate manner in breast cancer cells. 
A

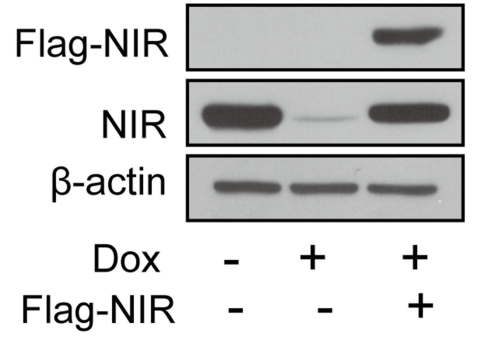

C
B MDA-MB231-Tet-on-shNIR

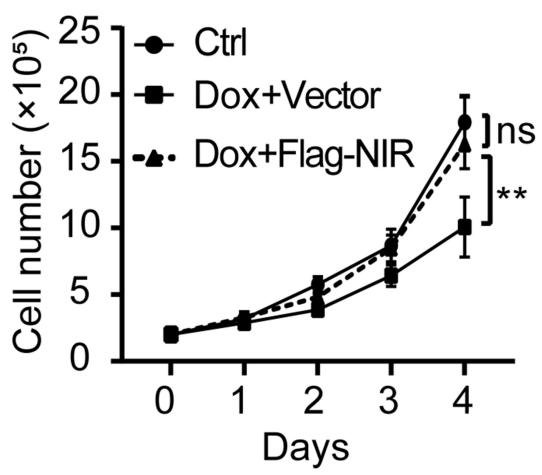

D

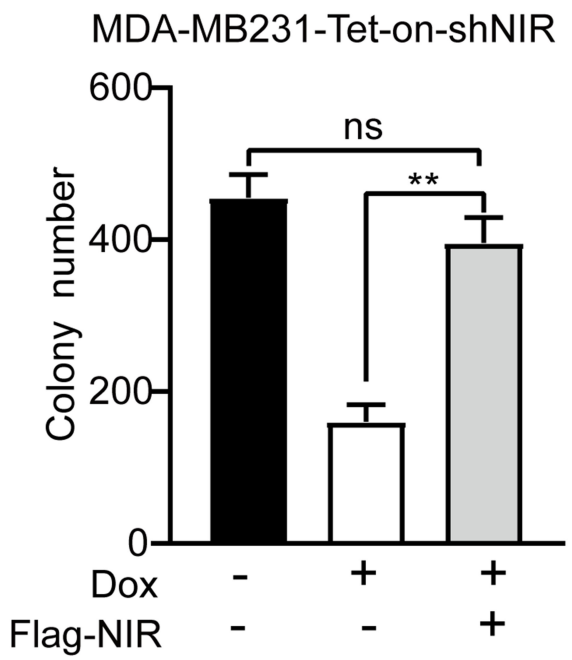

Flag-NIR
MDA-MB231-Tet-on-shNIR

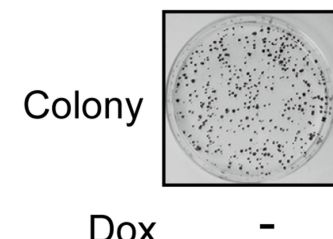

Flag-NIR

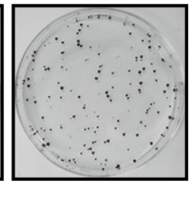

$+$

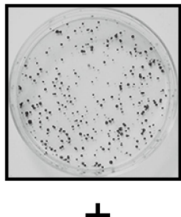

$+$

Figure 3 Re-expression NIR rescues the cell proliferation in NIR-silenced MDA-MB23I cells.

Notes: (A) NIR was re-expressed in NIR-silenced MDA-MB23I-Tet-on cells. Western blot results showed the NIR protein level was recovered in endogenous NIR-silenced MDA-MB23 I cells. Re-expression of NIR restored the proliferation (B) and cell colony formation (C and D) abilities in MDA-MB23I-Tet-on-shNIR cells. The cell colony was stained with Giemsa before counting. The proliferation data were performed by two-way ANOVA and the colony data were performed by one-way ANOVA for statistical analysis. Error bar represents the standard deviation from three independent experiments, **Denotes $p<0.01$; ns as no significant difference.

\section{EZH2 Recruits NIR to the Promoter Vicinity of FOXO3}

FOXO3 plays a pivotal function in cell proliferation. ${ }^{18}$ Since EZH2 has been shown to associate with the promoter vicinity of $\mathrm{FOXO} 3,{ }^{20,21}$ and direct interaction between NIR and EZH2 was uncovered in this study, we employed ChIP assays to investigate whether NIR and EZH2 co-occupied at the promoter vicinity of FOXO3 in MDA-MB231-HANIR cells. The results showed that both NIR and EZH2 were enriched at the promoter vicinity of FOXO3 (Figure 6AC). In agreement with these results, $\mathrm{H} 3 \mathrm{~K} 27 \mathrm{me} 3$ was detected at the promoter of FOXO3 (Figure 6D). Strikingly, H3K27ac, an active histone modification, was also detected at the FOXO3 promoter region (Figure 6E). The results indicate that two antagonistic modifications of $\mathrm{H} 3 \mathrm{~K} 27$ at the $\mathrm{FOXO} 3$ promoter region are intricately regulated in MDA-MB231 cells to control the expression of FOXO3.

After demonstrating the co-occupancy of NIR and EZH2 at the promoter vicinity of $\mathrm{FOXO} 3$, we next investigated the order of NIR and PRC2 recruitment to chromatin. Results from ChIP assays showed that silencing of EZH2 did not affect the protein levels of NIR and HA-tagged NIR (Figure 7A), but the silencing of EZH2 decreased the recruitment of NIR to the promoter vicinity of FOXO3 in MDA-MB231HA-NIR cells (Figure 7B). These results suggested that the recruitment of NIR to FOXO3 promoter region is EZH2dependent. In contrast, recruitment of EZH2 to the target gene was independent of NIR protein, as shown by ChIP assay in MDA-MB231-Tet-on-shNIR (Dox \pm ) cells (Figure $7 \mathrm{C}$ and D). Taken together, these findings suggest that NIR is 
A

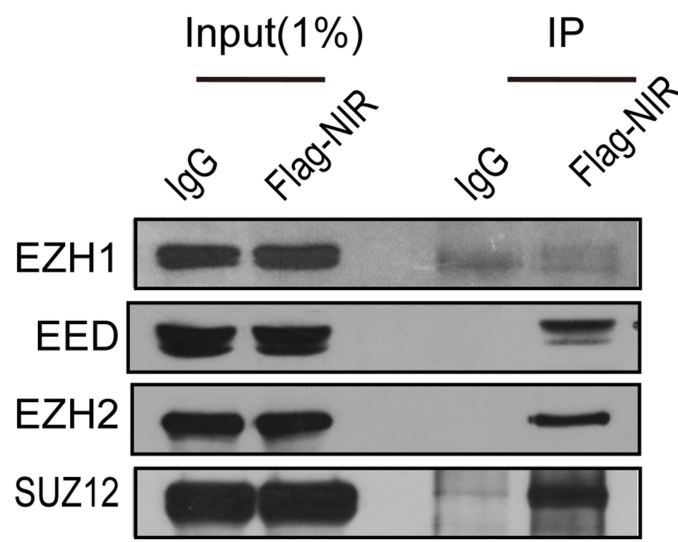

B

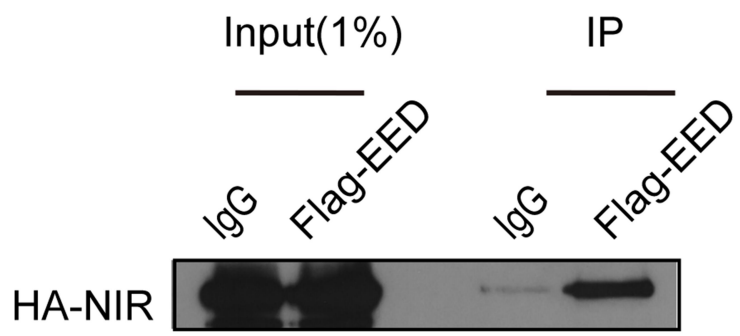

C

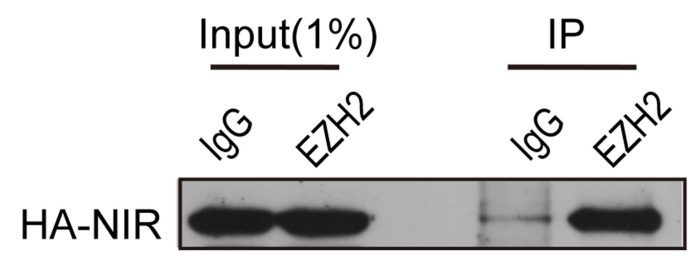

D

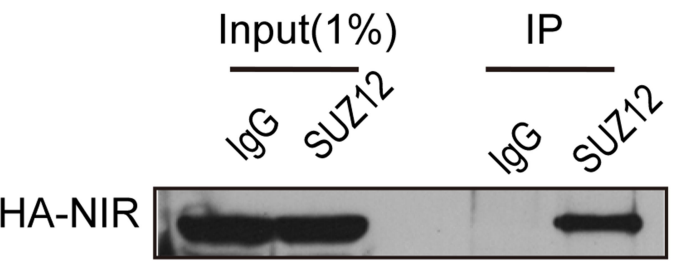

E

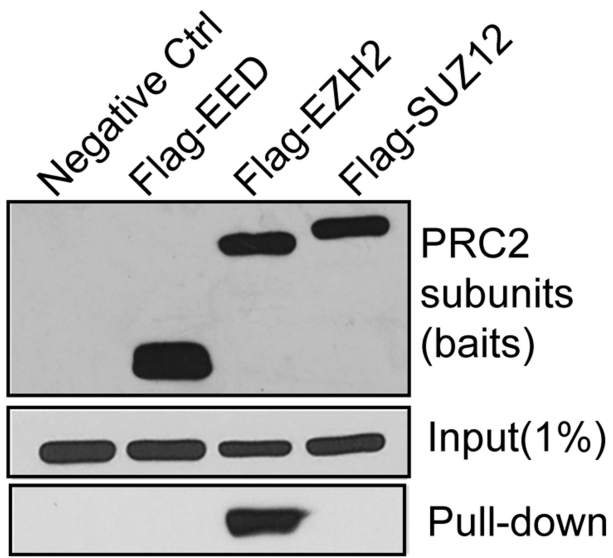

Figure 4 NIR directly associates with the PRC2 complex via EZH2.

Notes: (A) NIR-interacted proteins were co-immunoprecipitated (Co-IP) using Flag-NIR re-expressed MDA-MB23I-shNIR cell lysates. Anti-Flag antibody was used for CoIP and irrelevant IgG was used as negative control. Western blot results showed that EZH2, SUZI2, and EED were specifically immunoprecipitated by anti-Flag antibody, but EZHI. (B) NIR was specifically immunoprecipitated by Flag-tagged EED in MDA-MB23I-HANIR-FlagEED cells. (C and D) NIR was specifically immunoprecipitated by EZH2 (C), and SUZI 2 antibodies (D) in MDA-MB23I-HANIR cells. (E) Identification of PRC2 subunit directly interacting with NIR. Flag-tagged PRC2 subunits (EED, EZH2 and SUZI2) were in-vitro translated to serve as immobilized-baits and the amounts of baits were normalized by anti-flag Western blot. The same amount of in vitro translated HA-NIR was served as input. Immunoprecipitation assay showed Flag-EZH2 could specifically interact with NIR, but EED or SUZI 2.

recruited to the specific chromatin region by the PRC2 complex via direct interaction with EZH2.

\section{NIR Regulates the Status of H3K27 Modifications}

Previous studies have shown that EZH2 suppresses FOXO3 transcription by regulating the level of $\mathrm{H} 3 \mathrm{~K} 27 \mathrm{me} 3$ and recruitment of DNMTs to the promoter region. ${ }^{20}$ Our results showed that both EZH2 and NIR were recruited to the promoter vicinity of FOXO3 which encompass both histone H3K27 acetylation and trimethylation, indicating that NIR might modulate PRC2 function via regulating H3K27 modifications. To test this hypothesis, we performed ChIP assays using anti-H3K27me3 and anti-H3K27ac antibodies in Dox- inducible MDA-MB231-Tet-on-shNIR cells, with and without Dox treatment. The results showed that silencing NIR increased the level of H3K27ac and decreased H3K27 me3 at the $\mathrm{FOXO} 3$ promoter region (Figure 8). These results revealed the mechanism by which NIR suppresses FOXO3 transcription through balancing $\mathrm{H} 3 \mathrm{~K} 27 \mathrm{me} 3$ and $\mathrm{H} 3 \mathrm{~K} 27 \mathrm{ac}$ levels at the $\mathrm{FOXO} 3$ promoter region.

\section{NIR Regulates Cell Proliferation via Controlling the Expression of FOXO3}

Since our data showed that depletion of NIR enhanced FOXO3 expression while suppressing cell proliferation in MDA-MB231 cells, and FOXO3 has been shown to regulate cell growth. ${ }^{19}$ We investigated whether NIR 
A

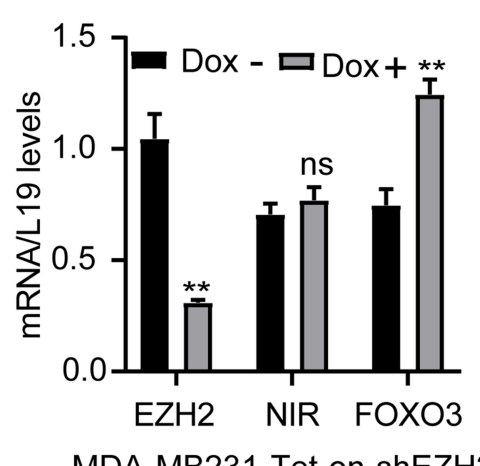

C

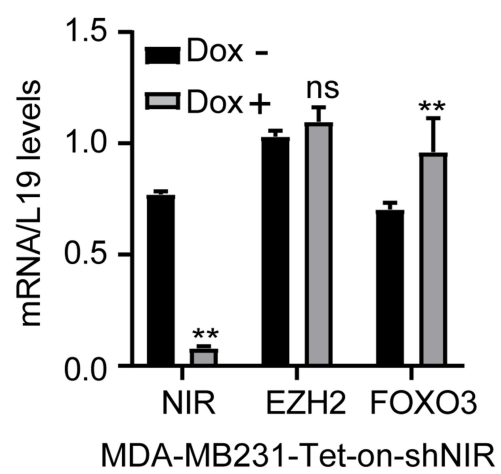

E

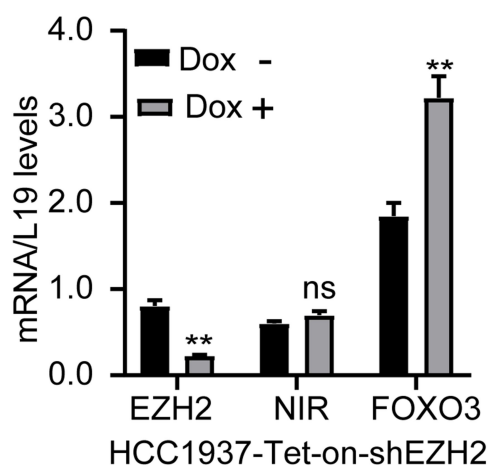

G

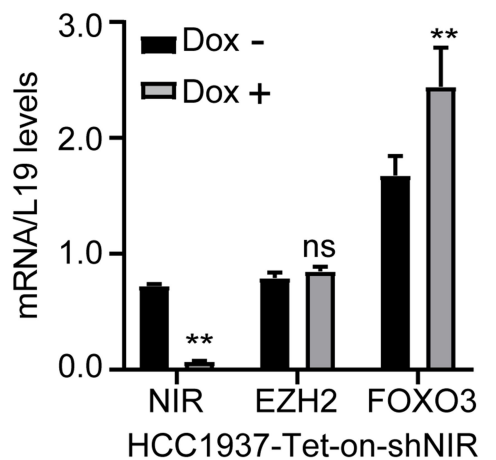

B
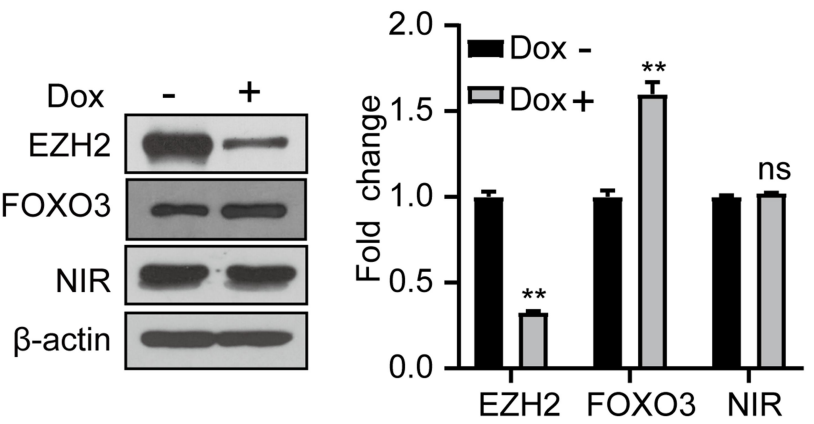

MDA-MB231-Tet-on-shEZH2

D
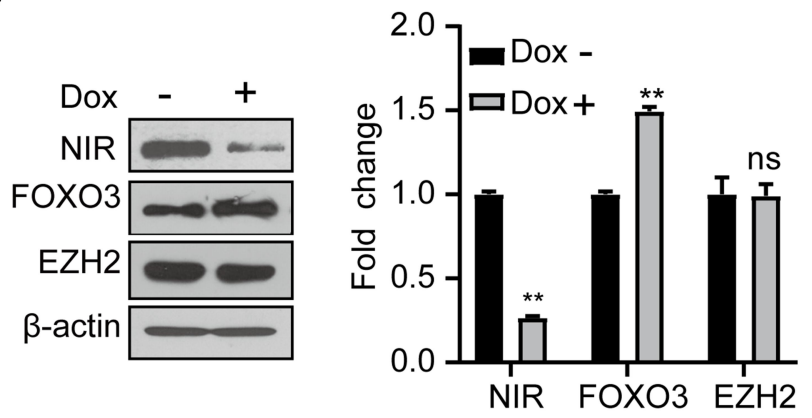

MDA-MB231-Tet-on-shNIR

$\mathbf{F}$
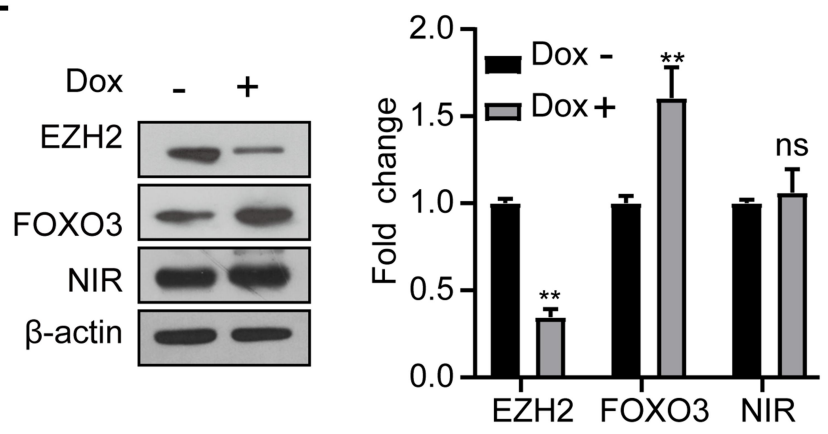

HCC1937-Tet-on-shEZH2

H
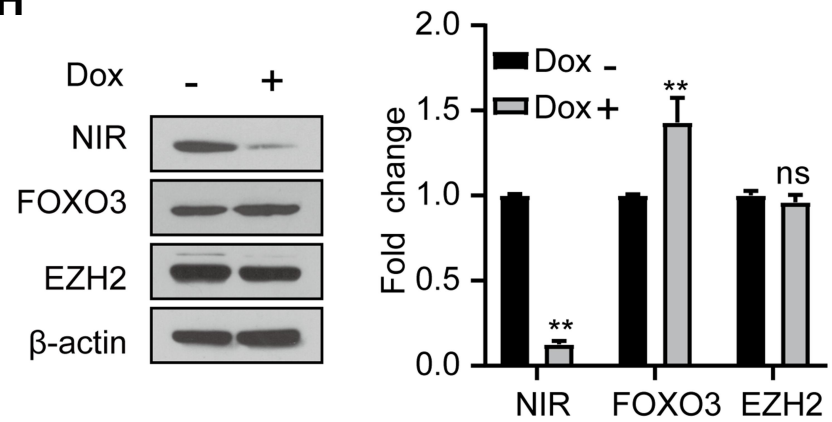

HCC1937-Tet-on-shNIR

Figure $5 \mathrm{EZH} 2$ and NIR co-regulate $\mathrm{FOXO} 3$ expression in breast cancer cells.

Notes: Silencing EZH2 increased the FOXO3 mRNA and protein level but did not affect NIR expression (A and B). The expression of EZH2 was not regulated by NIR, while the FOXO3 mRNA and protein levels were increased in NIR-silenced MDA-MB23I-Tet-on cells (C and D). The expression of FOXO3 was moderately increased, when EZH2 or NIR was silenced in HCCI 937 cells $(E-H)$. The RT-qPCR data of the indicated genes were normalized with LI9 mRNA levels and presented as mean \pm SD from three independent experiments, analyzed by two-way ANOVA test, ${ }^{* *} \mathrm{p}<0.0 \mathrm{I}$, ns as no significant difference. 
A

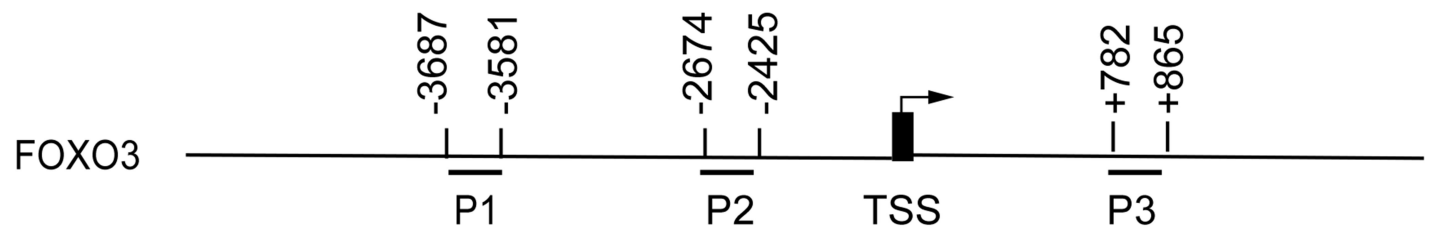

B

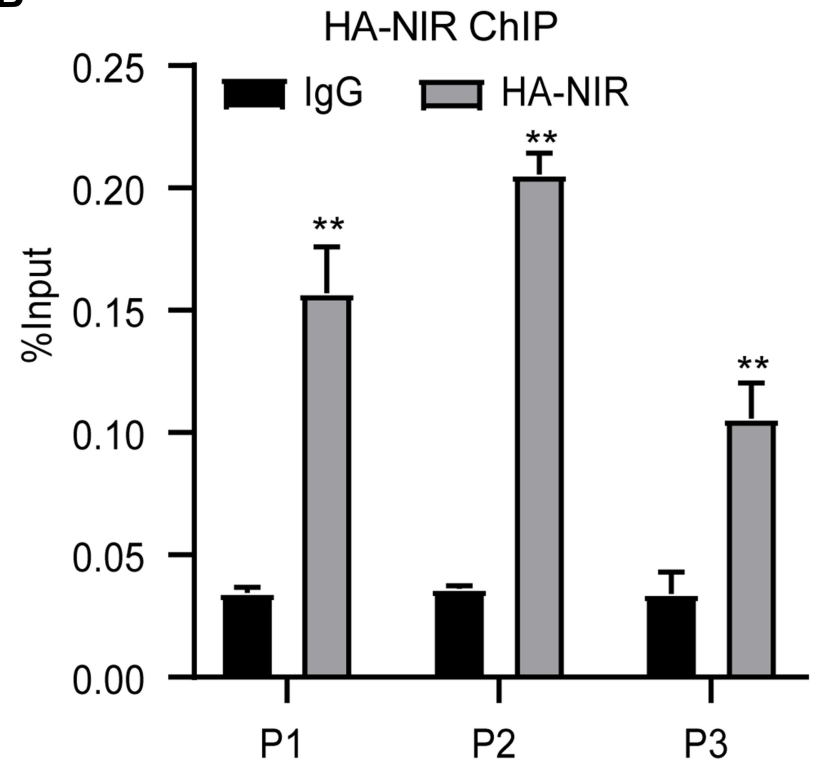

D

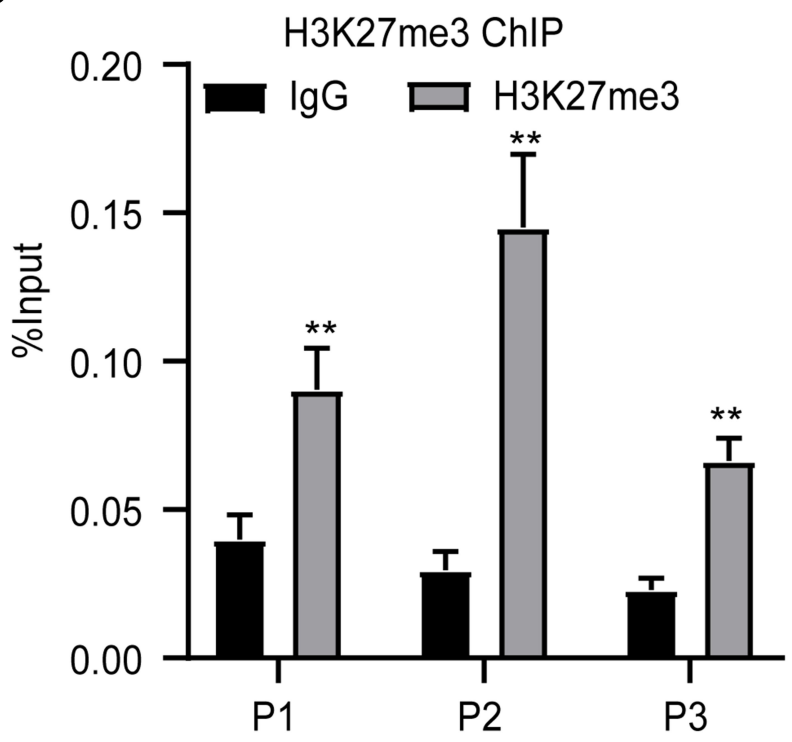

C

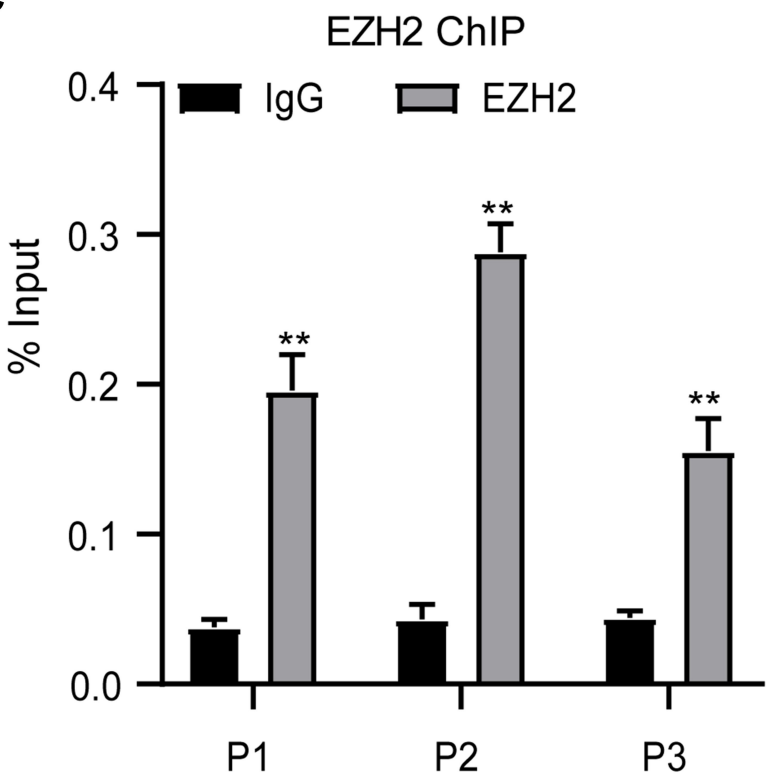

E

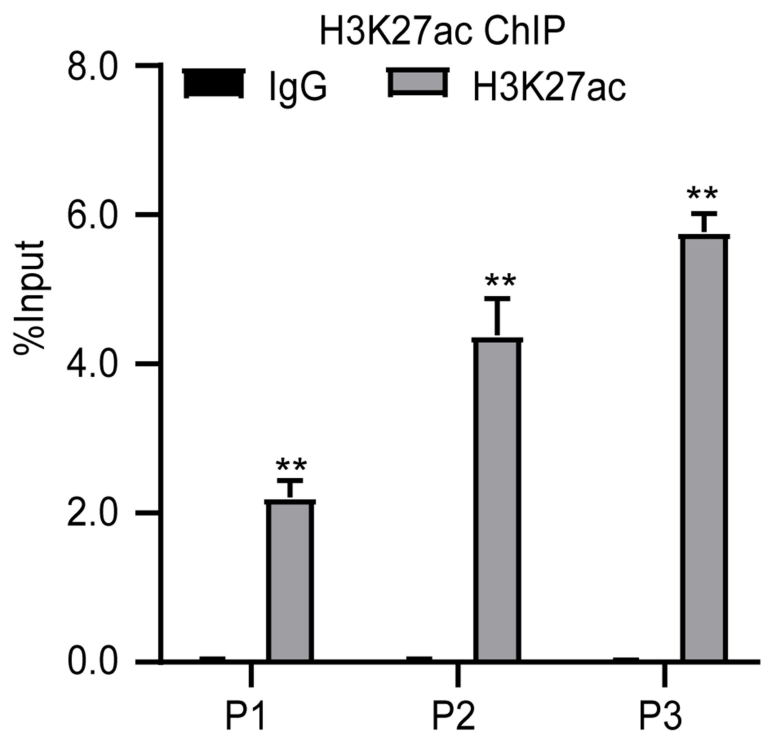

Figure 6 NIR, EZH2, H3K27me3, and H3K27ac are occupied at genomic loci of FOXO3.

Notes: (A) A schematic representation of the location of primers for ChIP-qPCR at FOXO3 genomic loci. (B and C) ChIP results showed that NIR and EZH2 had similar binding patterns at the vicinity of the promoter region of FOXO3, in MDA-MB23I-HANIR cells. (D) Similar to the EZH2 chromatin binding pattern, H3K27me3 was also enriched at the FOXO3 promoter region. (E) ChIP assay showed H3K27ac deposition at the FOXO3 genomic loci. The results were normalized to the amount of the input. Rabbit IgG was used as a negative control. All experiments were independently repeated three times, the results were presented as mean \pm SD, $* * D e n o t e s ~ P<0.0$. 
A
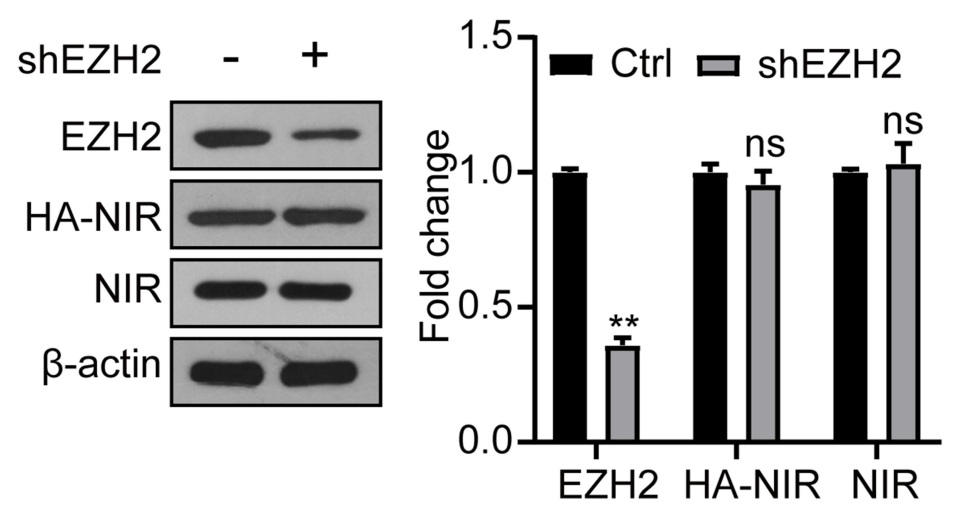

MDA-MB231-HANIR
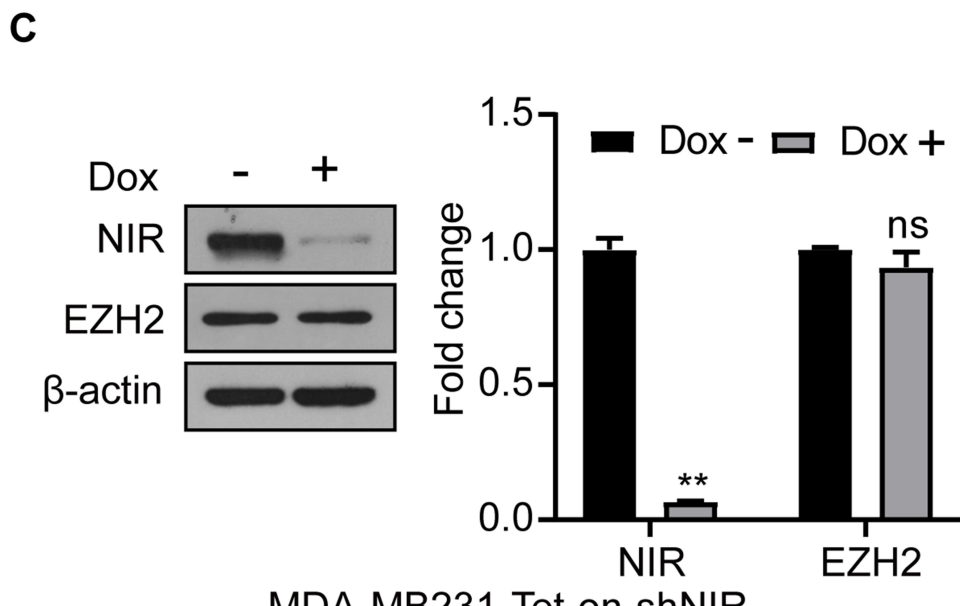

B

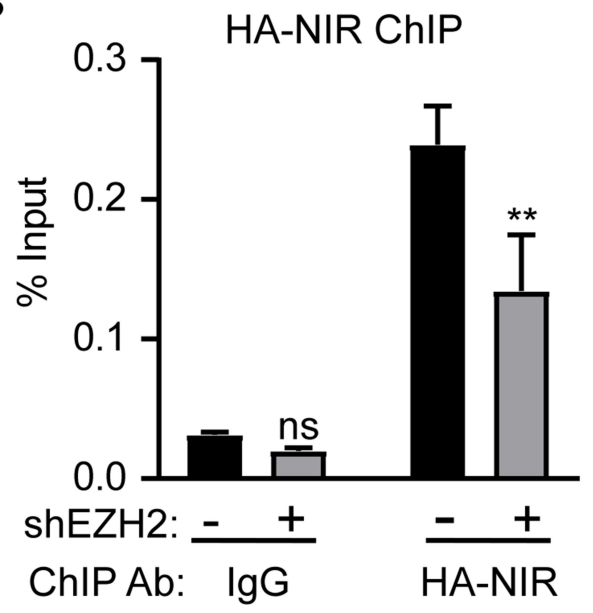

D

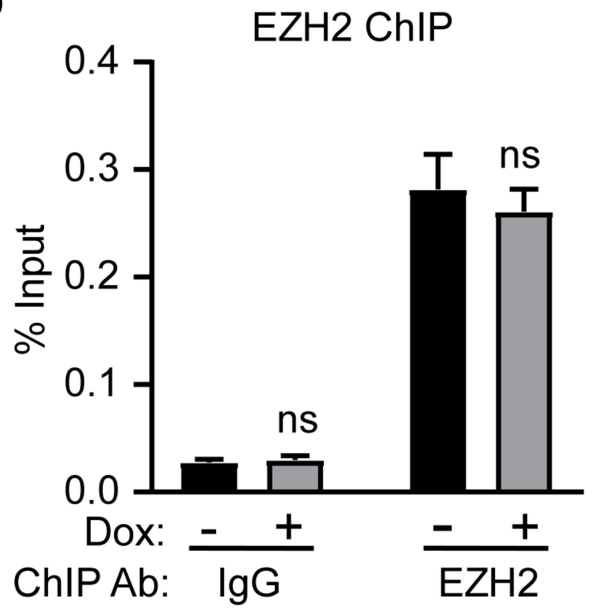

Figure 7 EZH2 recruits NIR to the vicinity promoter of FOXO3.

Notes: (A) EZH2 was silenced in MDA-MB23I-HANIR cells by infecting with pLVTHM-shEZH2 virus, the control cell was infected with pLVTHM-shGFP virus. The expression levels of NIR and HA-tagged NIR were not affected by silencing of EZH2. (B) Silencing of EZH2 decreased the binding of NIR at the FOXO3 promoter region. (C and D) The deposition of EZH2 at the FOXO3 promoter region was not influenced in NIR-silenced MDA-MB23I-Tet-on cells. Rabbit IgG was used as ChIP antibody negative control. The primer pair (P2) located in 2425bp upstream of FOXO3 TSS was used for ChIP-qPCR. All experiments were independently repeated three times. The results were normalized by input and presented as mean \pm SD and the two-way ANOVA test was performed for statistical analysis, $* * p<0.01$, ns as no significant difference.

regulated cell proliferation via FOXO3 in breast cancer cells. Indeed, we observed that the enhanced proliferation of MDA-MB231 cells caused by overexpression of NIR can be reversed by ectopic FOXO3 expression (Figure 9A and B). Moreover, the suppressed proliferation caused by NIR knockdown was restored by silencing of FOXO3 (Figure 9C and D). These results support that NIR modulates the proliferation of breast cancer cells, at least in part, in a FOXO3-dependent manner.

\section{Discussion}

In the present study, we found that high mRNA levels of NIR correlated with poor survival in breast cancer patients, and knockdown of NIR suppressed cellular proliferation and colony formation of breast cancer cells, which could be rescued by restoring the level of NIR. While NIR was reported to modulate the transcription activity of $\mathrm{p} 53,{ }^{4}$ the NIR regulatory function of cell proliferation was observed in p53-mutant cell lines, revealing a p53-independent mechanism of NIR function in breast cancer cells. We discovered 
A

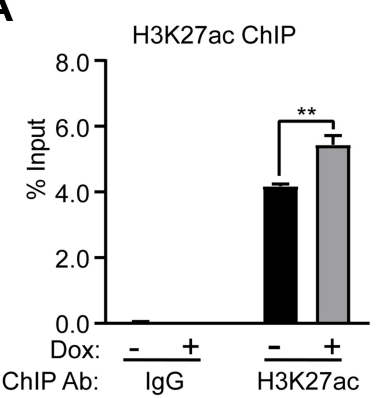

B

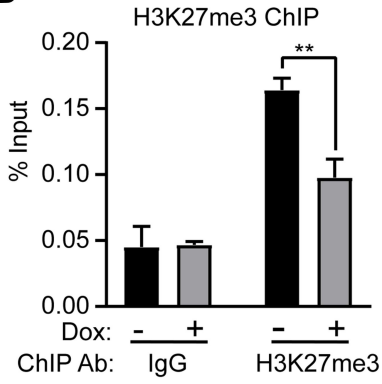

Figure 8 NIR modulates the modification of $\mathrm{H} 3 \mathrm{~K} 27$ at the $\mathrm{FOXO} 3$ promoter region.

Notes: ChIP assays were performed by using anti-H3K27ac and anti-H3K27me3 antibodies in MDA-MB23I-Tet-on-shNIR cells. The results showed that silencing of NIR enhanced H3K27ac enrichment (A) and decreased H3K27me3 deposition (B) at the $\mathrm{FOXO} 3$ promoter region. $\lg \mathrm{G}$ was used as control and the data were normalized to input. The primer pair (P2) located in 2425bp upstream of FOXO3 TSS was used for ChIP-qPCR. Two-way ANOVA test was performed, the bars represented the mean $\pm \mathrm{SD}$ from three independent experiments, **P $<0.01$.

that overexpressing NIR represses the expression of FOXO3, a tumor suppressor gene in breast cancer cells. Moreover, NIR was recruited to $\mathrm{FOXO} 3$ promoter via

interacting with EZH2 in the PRC2 complex to modulate the H3K27ac and H3K27me3 levels. The FOXO3 activation accounts for inhibition of breast cancer cell proliferation in NIR-silenced breast cancer cells.

$\mathrm{EZH} 2$, the catalyzing core of PRC2, is often found to suppress tumor suppressor genes in tumorigenesis. ${ }^{13}$ In addition to the core subunits of EED and SUZ12 that were required for the catalyzing activity of EZH2 in PRC2, a variety of PRC2 interaction proteins had been identified for modulation of PRC2 functions. ${ }^{11,28,29}$ For example, the Polycomb-like protein PHF19 can interact with PRC2 in prostate cancer cells, and depletion of PHF19 increases PRC2 occupancy and H3K27me3 deposition on PRC2 associated chromatin regions. ${ }^{30}$ SETDB1, a histone methyltransferase of $\mathrm{H} 3 \mathrm{~K} 9$, was shown to associate with PRC2-interacting protein JARID2 to modulate EZH2 and H3K27me3 distribution on the PRC2 target genes. ${ }^{31}$ Our reciprocal coimmunoprecipitation assays confirmed the association between NIR and PRC2 complex. EZH2, EED, and SUZ12 - but not EZH1 could be immunoprecipitated by Flag-NIR,
A

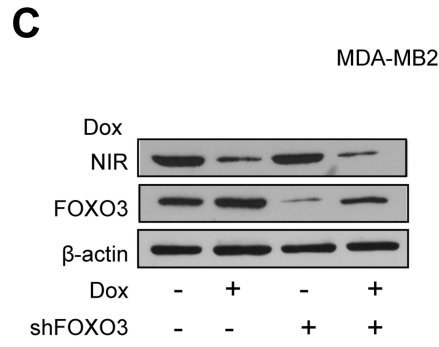

MDA-MB231

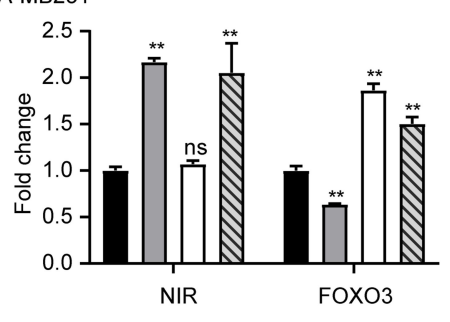

- Ctrl+Ctrl 口 Ctrl+Flag-FOXO3 口HA-NIR+Ctrl $\square$ HA-NIR+Flag-FOXO3

B

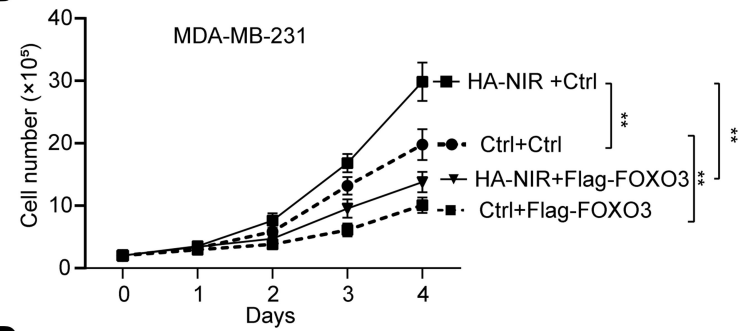

D

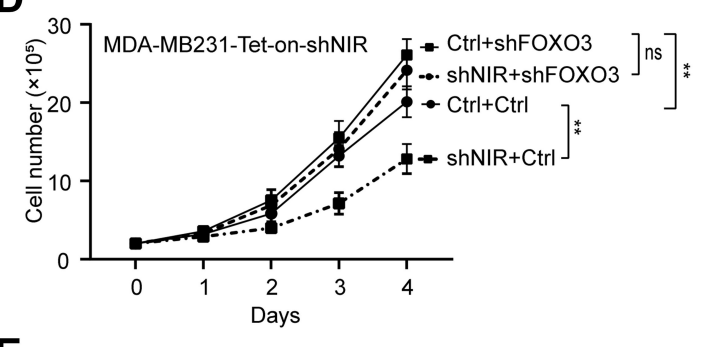

E

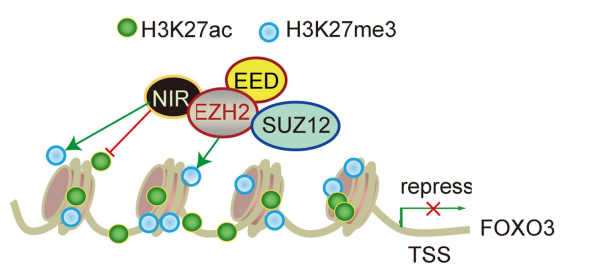

Figure 9 NIR regulates breast cancer cell proliferation via FOXO3.

Notes: (A and B) Overexpression of FOXO3 attenuated the promoted cell proliferation, which was induced by overexpression of NIR in MDA-MB23I cells. (C and D) Silencing of FOXO3 significantly rescued the cell proliferation repression induced by the knockdown of NIR in MDA-MB23I cells. All experiments were repeated three times independently. The results were performed by two-way ANOVA test and showed as mean $\pm \mathrm{SD}$, **p $<0.0 \mathrm{l}$; ns as no significant difference. (E) Graphic model of how NIR regulates FOXO3 transcription. NIR interacts with PRC2 by bridging EZH2, and NIR contributes to PRC2 inhibition function by inhibition of H3K27ac marker and enhancing of $\mathrm{H} 3 \mathrm{~K} 27 \mathrm{me} 3$ marker at the promoter region of FOXO3. 
and NIR could be co-precipitated with PRC2 core subunits using anti-EZH2, -FlagEED, or -SUZ12 antibodies. These results identified NIR as an interacting protein of the PRC2 complex. The previous report has shown that the PRC2 complex could recruit DNMTs or HDACs to enhance its repression function. ${ }^{32}$ Because NIR is known to inhibit histone acetylation, we surmised that NIR may enhance the function of PRC2 via modulation of histone modifications. We confirmed that FOXO3 is co-regulated by NIR and EZH2, and that EZH2, NIR, H3K27me3, and H3K27ac share a similar pattern of enrichment at the promoter region of FOXO3. It has been reported that recruitment of PRC2 to the genomic loci could be regulated by lncRNAs such as HOTAIR and XIST and YY1, the mammalian ortholog of the Drosophila PRE DNAbinding protein PHO. ${ }^{33-36}$ We found that knockdown of NIR did not affect the recruitment of EZH2 at the $\mathrm{FOXO} 3$ promoter region, whereas silencing EZH2 led to decreased NIR enrichment at the $\mathrm{FOXO} 3$ promoter region. These results implicated that NIR is a downstream effector of EZH2 to regulate H3K27me3 level on PRC2-target genes.

The status of acetylation and methylation of the histone tails has a crucial role in regulating chromatin structure and gene expression. ${ }^{37}$ The amino group of lysine 27 of histone $\mathrm{H} 3$ could be subjected to either methylation or acetylation, which have an opposing effect on gene transcription. The H3K27me3 catalyzed by PRC2 is a repressive mark, while the $\mathrm{H} 3 \mathrm{~K} 27 \mathrm{ac}$ mark is generally associated with activated genes. ${ }^{11}$ It has been reported that the H3K27me3 mark is associated with temporarily repressed gene expression, which could be dynamically changed depending on the cell contexts. ${ }^{38} \mathrm{H} 3 \mathrm{~K} 27$ acetylation and S28 phosphorylation are directly coupled, and such combination can functionally antagonize PRC2-mediated H3K27me3 mark. ${ }^{39}$ Using a NIR inducible knockdown cell line, we found that silencing of NIR resulted in increased $\mathrm{H} 3 \mathrm{~K} 27 \mathrm{ac}$ and reduction of $\mathrm{H} 3 \mathrm{~K} 27 \mathrm{me} 3$ level at the promoter region of FOXO3, implying the intricated balance of $\mathrm{H} 3 \mathrm{~K} 27 \mathrm{ac}$ and H3K27me3 levels by NIR through modulating PRC2 activity. Since NIR has INHAT (inhibitor of histone acetyltransferase) activity, the interaction of NIR and EZH2 may block the accessibility of acetyltransferases to catalyze $\mathrm{H} 3 \mathrm{~K} 27 \mathrm{ac}$, while facilitating PRC2 to catalyze the H3K27me3 mark.

FOXO3, as a central transcription factor, can regulate multiple cellular processes including cell proliferation. ${ }^{40}$ The hypermethylation of FOXO3 gene promoter and low expression of FOXO3 is associated with a higher tumor grade and poor survival in breast cancer. ${ }^{20}$ FOXO3 is negatively regulated by EZH2 via the H3K27me3-mediated silencing mechanism and in hepatocellular carcinoma. ${ }^{21}$ At least in breast cancer cell lines, knocking down NIR increased FOXO3 expression at both mRNA and protein levels and this regulation appears to depend on $\mathrm{H} 3 \mathrm{~K} 27$ modification near FOXO3 promoter manifested by NIR. The FOXO3 mediated growth inhibition is attributed to $\mathrm{FOXO} 3$ downstream target genes, including CDK inhibitors (p21 Cip1 p27 Kip1) and cell cycle-related genes (cyclin D1/D2). ${ }^{41-43}$ Our results demonstrated that NIR promotes breast cancer cell proliferation through repressing of FOXO3, as the reduced cell proliferation upon NIR knockdown could be effectively rescued by silencing FOXO3. The ectopic expression of either FOXO3 or NIR antagonizes each other in the regulation of cell proliferation. Collectively, like the mode schematic shown in Figure 9E, our data reveal a novel mechanism by which NIR regulates breast cancer cell proliferation through $\mathrm{FOXO} 3$ and uncovers the cooperative action of NIR with PRC2-EZH2 to repress FOXO3 expression via regulation of histone $\mathrm{H} 3 \mathrm{~K} 27$ modifications.

\section{Conclusion}

Our study demonstrated that a high level of NIR expression was associated with poor prognosis in breast cancer patients. Knockdown of NIR suppresses the proliferation of breast cancer cells by improving FOXO3. Therefore, NIR might be explored as a new therapeutic target for breast cancer.

\section{Acknowledgments}

Lentivirus expression vectors are kind gifts from Dr Trono Didier (University of Geneva). This work was supported by the National Natural Science Foundation of China (NO. 31360273, 81360314), and Natural Science Foundation of Guangxi (No. 2014GXNSFAA118278).

\section{Disclosure}

The authors proclaim no conflict of interests.

\section{References}

1. Bray F, Ferlay J, Soerjomataram I, Siegel RL, Torre LA, Jemal A. Global cancer statistics 2018: GLOBOCAN estimates of incidence and mortality worldwide for 36 cancers in 185 countries. CA Cancer J Clin. 2018;68(6):394-424. doi:10.3322/caac.21492

2. Harbeck N, Gnant M. Breast cancer. Lancet. 2017;389 (10074):1134-1150. doi:10.1016/S0140-6736(16)31891-8

3. Lee EY, Muller WJ. Oncogenes and tumor suppressor genes. Cold Spring Harb Perspect Biol. 2010;2(10):a003236. doi:10.1101/cshperspect.a003236 
4. Hublitz P, Kunowska N, Mayer UP, et al. NIR is a novel INHAT repressor that modulates the transcriptional activity of p53. Genes Dev. 2005;19(23):2912-2924. doi:10.1101/gad.351205

5. Duteil D, Tourrette Y, Eberlin A, et al. The histone acetyltransferase inhibitor Nir regulates epidermis development. Development. 2018;145(6):dev158543. doi:10.1242/dev.158543

6. Heyne K, Willnecker V, Schneider J, et al. NIR, an inhibitor of histone acetyltransferases, regulates transcription factor TAp63 and is controlled by the cell cycle. Nucleic Acids Res. 2010;38 (10):3159-3171. doi:10.1093/nar/gkq016

7. Ma CA, Pusso A, Wu L, et al. Novel INHAT repressor (NIR) is required for early lymphocyte development. Proc Natl Acad Sci U S A. 2014;111(38):13930-13935. doi:10.1073/pnas.1310118111

8. Jones PA, Baylin SB. The epigenomics of cancer. Cell. 2007;128 (4):683-692. doi:10.1016/j.cell.2007.01.029

9. Simon JA, Lange CA. Roles of the EZH2 histone methyltransferase in cancer epigenetics. Mutat Res. 2008;647(1-2):21-29. doi:10.1016/ j.mrfmmm.2008.07.010

10. Comet I, Riising EM, Leblanc B, Helin K. Maintaining cell identity: PRC2-mediated regulation of transcription and cancer. Nat Rev Cancer. 2016;16(12):803-810. doi:10.1038/nrc.2016.83

11. Pasini D, Malatesta M, Jung HR, et al. Characterization of an antagonistic switch between histone H3 lysine 27 methylation and acetylation in the transcriptional regulation of Polycomb group target genes. Nucleic Acids Res. 2010;38(15):4958-4969. doi:10.1093/nar/gkq244

12. Kondo Y, Shen L, Cheng AS, et al. Gene silencing in cancer by histone $\mathrm{H} 3$ lysine 27 trimethylation independent of promoter DNA methylation. Nat Genet. 2008;40(6):741-750. doi:10.1038/ng.159

13. Duan R, Du W, Guo W. EZH2: a novel target for cancer treatment. J Hematol Oncol. 2020;13(1):104. doi:10.1186/s13045-020-00937-8

14. Ernst T, Chase AJ, Score J, et al. Inactivating mutations of the histone methyltransferase gene EZH2 in myeloid disorders. Nat Genet. 2010;42(8):722-726. doi:10.1038/ng.621

15. Chen S, Bohrer LR, Rai AN, et al. Cyclin-dependent kinases regulate epigenetic gene silencing through phosphorylation of EZH2. Nat Cell Biol. 2010;12(11):1108-1114. doi:10.1038/ncb2116

16. Wei Y, Chen YH, Li LY, et al. CDK1-dependent phosphorylation of EZH2 suppresses methylation of $\mathrm{H} 3 \mathrm{~K} 27$ and promotes osteogenic differentiation of human mesenchymal stem cells. Nat Cell Biol. 2011;13(1):87-94. doi:10.1038/ncb2139

17. Wan J, Zhan J, Li S, et al. PCAF-primed EZH2 acetylation regulates its stability and promotes lung adenocarcinoma progression. Nucleic Acids Res. 2015;43(7):3591-3604. doi:10.1093/nar/gkv238

18. Liu Y, Ao X, Ding W, et al. Critical role of FOXO3a in carcinogenesis. Mol Cancer. 2018;17(1). doi:10.1186/s12943-0180856-3

19. Liu H, Song Y, Qiu H, et al. Downregulation of FOXO3a by DNMT1 promotes breast cancer stem cell properties and tumorigenesis. Cell Death Differ. 2020;27(3):966-983. doi:10.1038/s41418-019-0389-3

20. Gong $\mathrm{C}$, Yao S, Gomes AR, et al. BRCA1 positively regulates FOXO3 expression by restricting $\mathrm{FOXO} 3$ gene methylation and epigenetic silencing through targeting EZH2 in breast cancer. Oncogenesis. 2016;5:e214. doi:10.1038/oncsis.2016.23

21. Gao SB, Xu B, Ding LH, et al. The functional and mechanistic relatedness of EZH2 and menin in hepatocellular carcinoma. $J$ Hepatol. 2014;61(4):832-839. doi:10.1016/j.jhep.2014.0 5.015

22. Wiznerowicz M, Trono D. Conditional suppression of cellular genes: lentivirus vector-mediated drug-inducible RNA interference. $J$ Virol. 2003;77(16):8957-8961. doi:10.1128/jvi.77.16.8957-8951.2003

23. Lee TI, Johnstone SE, Young RA. Chromatin immunoprecipitation and microarray-based analysis of protein location. Nat Protoc. 2006;1 (2):729-748. doi:10.1038/nprot.2006.98
24. Heyne K, Forster J, Schule R, Roemer K. Transcriptional repressor NIR interacts with the p53-inhibiting ubiquitin ligase MDM2. Nucleic Acids Res. 2014;42(6):3565-3579. doi:10.1093/nar/gkt1 371

25. Curtis C, Shah SP, Chin SF, et al. The genomic and transcriptomic architecture of 2000 breast tumours reveals novel subgroups. Nature. 2012;486(7403):346-352. doi:10.1038/nature10983

26. Laugesen A, Hojfeldt JW, Helin K. Molecular mechanisms directing PRC2 recruitment and H3K27 methylation. Mol Cell. 2019;74 (1):8-18. doi:10.1016/j.molcel.2019.03.011

27. Han L, Zhang HC, Li L, Li CX, Di X, Qu X. Downregulation of long noncoding RNA HOTAIR and EZH2 induces apoptosis and inhibits proliferation, invasion, and migration of human breast cancer cells. Cancer Biother Radiopharm. 2018;33(6):241-251. doi:10.1089/ cbr.2017.2432

28. Ueda T, Sanada M, Matsui H, et al. EED mutants impair polycomb repressive complex 2 in myelodysplastic syndrome and related neoplasms. Leukemia. 2012;26(12):2557-2560. doi:10.1038/ leu.2012.146

29. Pasini D, Bracken AP, Jensen MR, Lazzerini Denchi E, Helin K. Suz12 is essential for mouse development and for EZH2 histone methyltransferase activity. EMBO J. 2004;23(20):4061-4071. doi:10.1038/sj.emboj.7600402

30. Jain P, Ballare C, Blanco E, Vizan P, Di Croce L. PHF19 mediated regulation of proliferation and invasiveness in prostate cancer cells. Elife. 2020;9. doi:10.7554/eLife.51373.

31. Fei Q, Yang X, Jiang H, et al. SETDB1 modulates PRC2 activity at developmental genes independently of $\mathrm{H} 3 \mathrm{~K} 9$ trimethylation in mouse ES cells. Genome Res. 2015;25(9):1325-1335. doi:10.1101/ gr. 177576.114

32. Vire E, Brenner C, Deplus R, et al. The Polycomb group protein EZH2 directly controls DNA methylation. Nature. 2006;439 (7078):871-874. doi:10.1038/nature04431

33. Rinn JL, Kertesz M, Wang JK, et al. Functional demarcation of active and silent chromatin domains in human HOX loci by noncoding RNAs. Cell. 2007;129(7):1311-1323. doi:10.1016/j. cell.2007.05.022

34. Colognori D, Sunwoo H, Kriz AJ, Wang CY, Lee JT. Xist deletional analysis reveals an interdependency between Xist RNA and polycomb complexes for spreading along the inactive X. Mol Cell. 2019;74(1):101-117 e10. doi:10.1016/j.molcel.2019.01.015

35. Wilkinson FH, Park K, Atchison ML. Polycomb recruitment to DNA in vivo by the YY1 REPO domain. Proc Natl Acad Sci U S A. 2006;103(51):19296-19301. doi:10.1073/pnas.0603564103

36. Xi H, Yu Y, Fu Y, Foley J, Halees A, Weng Z. Analysis of overrepresented motifs in human core promoters reveals dual regulatory roles of YY1. Genome Res. 2007;17(6):798-806. doi:10.1101/gr.5754 707

37. Mohammad HP, Barbash O, Creasy CL. Targeting epigenetic modifications in cancer therapy: erasing the roadmap to cancer. Nat Med. 2019;25(3):403-418. doi:10.1038/s41591-019-0376-8

38. Holoch D, Margueron R. Mechanisms regulating PRC2 recruitment and enzymatic activity. Trends Biochem Sci. 2017;42(7):531-542. doi:10.1016/j.tibs.2017.04.003

39. Khan DH, Healy S, He S, et al. Mitogen-induced distinct epialleles are phosphorylated at either H3S10 or H3S28, depending on H3K27 acetylation. Mol Biol Cell. 2017;28(6):817-824. doi:10.1091/mbc. E16-08-0618

40. Stefanetti RJ, Voisin S, Russell A, Lamon S. Recent advances in understanding the role of FOXO3. F1000Res. 2018;7:1372. doi:10.12688/f1000research.15258.1

41. Hauck L, Harms C, Grothe D, et al. Critical role for FoxO3a-dependent regulation of $\mathrm{p} 21 \mathrm{CIP} 1 / \mathrm{WAF} 1$ in response to statin signaling in cardiac myocytes. Circ Res. 2007;100(1):50-60. doi:10.1161/01.RES.0000254704.92532.b9 
42. Chandramohan V, Mineva ND, Burke B, et al. c-Myc represses FOXO3a-mediated transcription of the gene encoding the p27 (Kip1) cyclin dependent kinase inhibitor. J Cell Biochem. 2008;104 (6):2091-2106. doi:10.1002/jcb.21765
43. Schmidt M, Fernandez de Mattos S, van der Horst A, et al. Cell cycle inhibition by FoxO forkhead transcription factors involves downregulation of cyclin D. Mol Cell Biol. 2002;22(22):7842-7852. doi: $10.1128 / \mathrm{mcb} .22 .22 .7842-7852.2002$

\section{Publish your work in this journal}

OncoTargets and Therapy is an international, peer-reviewed, open access journal focusing on the pathological basis of all cancers, potential targets for therapy and treatment protocols employed to improve the management of cancer patients. The journal also focuses on the impact of management programs and new therapeutic

Submit your manuscript here: https://www.dovepress.com/oncotargets-and-therapy-journal agents and protocols on patient perspectives such as quality of life, adherence and satisfaction. The manuscript management system is completely online and includes a very quick and fair peer-review system, which is all easy to use. Visit http://www.dovepress.com/ testimonials.php to read real quotes from published authors. 